\title{
BI-LAGRANGIAN STRUCTURES ON NILMANIFOLDS
}

\author{
M. J. D. HAMILTON
}

\begin{abstract}
We study bi-Lagrangian structures (a symplectic form with a pair of complementary Lagrangian foliations, also known as para-Kähler or Künneth structures) on nilmanifolds of dimension less than or equal to 6 . In particular, building on previous work of several authors, we determine which 6 -dimensional nilpotent Lie algebras admit a bi-Lagrangian structure. In dimension 6 , there are (up to isomorphism) 26 nilpotent Lie algebras which admit a symplectic form, 16 of which admit a bi-Lagrangian structure and 10 of which do not. We also calculate the curvature of the canonical connection of these bi-Lagrangian structures.
\end{abstract}

\section{INTRODUCTION}

In this article we are interested in bi-Lagrangian structures on smooth manifolds, defined as follows:

Definition 1.1. Let $M$ be a smooth manifold. Then a bi-Lagrangian structure consists of a symplectic form $\omega \in \Omega^{2}(M)$ and a pair $\mathcal{F}, \mathcal{G}$ of Lagrangian foliations on $M$ such that $T M=T \mathcal{F} \oplus T \mathcal{G}$. Bi-Lagrangian structures are also known as Künneth structures.

Recall that according to the Frobenius theorem a foliation $\mathcal{F}$ on a smooth manifold $M$ is given by a distribution $T \mathcal{F} \subset T M$ which is integrable in the sense that $[X, Y] \in \Gamma(T \mathcal{F})$ for all sections $X, Y \in \Gamma(T \mathcal{F})$. A foliation $\mathcal{F}$ on a symplectic manifold $\left(M^{2 n}, \omega\right)$ is called Lagrangian if $T \mathcal{F}$ is a Lagrangian subbundle of $(T M, \omega)$, i.e. the rank of $T \mathcal{F}$ is $n=\frac{1}{2} \operatorname{dim} M$ and $\omega(X, Y)=0$ for all $X, Y \in T \mathcal{F}$.

In the following we will consider foliations as integrable distributions and place less emphasis on foliation-specific aspects such as the global topology or the structure of leaves.

For background on bi-Lagrangian structures see, for instance, [5], [9], [13] and the forthcoming book [12]. Bi-Lagrangian structures appear frequently in other contexts of geometry, for example, hypersymplectic structures, introduced by Hitchin [14], and Anosov symplectomorphisms define bi-Lagrangian structures (see Chapters 5 and 8 in [12]).

Example 1.2. The simplest example of a bi-Lagrangian structure is given by $M=$ $\mathbb{R}^{2 n}=\mathbb{R}^{n} \times \mathbb{R}^{n}$ with coordinates $x_{1}, \ldots, x_{n}, y_{1}, \ldots, y_{n}$, symplectic form

$$
\omega_{0}=\sum_{i=1}^{n} d x_{i} \wedge d y_{i}
$$

Date: February 22, 2019. 
and Lagrangian foliations $\mathcal{F}_{0}$ and $\mathcal{G}_{0}$, whose leaves are given by the affine Lagrangian subspaces $\mathbb{R}^{n} \times\{*\}$ and $\{*\} \times \mathbb{R}^{n}$. This bi-Lagrangian structure descends to the torus $T^{2 n}$.

Non-trivial examples of bi-Lagrangian structures are usually difficult to construct. An interesting class of examples comes from nilmanifolds. We want to study left-invariant bi-Lagrangian structures on Lie groups $G$, which we can equivalently think of as the following linear structures on the Lie algebra $\mathfrak{g}$ of $G$ :

Definition 1.3. Let $\mathfrak{g}$ be a real Lie algebra. A symplectic form on $\mathfrak{g}$ is a closed and non-degenerate 2-form $\omega \in \Lambda^{2} \mathfrak{g}^{*}$ and a foliation on $\mathfrak{g}$ is a subalgebra $\mathcal{F} \subset \mathfrak{g}$. A bi-Lagrangian structure on $\mathfrak{g}$ consists of a symplectic form $\omega$ and two Lagrangian subalgebras $\mathcal{F}, \mathcal{G} \subset \mathfrak{g}$ such that $\mathfrak{g}=\mathcal{F} \oplus \mathcal{G}$ (vector space direct sum).

Bi-Lagrangian structures on Lie algebras in general have been studied, for example, in [1], [4] and [15].

In the case of a nilpotent Lie algebra $\mathfrak{g}$, the corresponding left-invariant biLagrangian structure on the associated, simply connected nilpotent Lie group $G$ induces a bi-Lagrangian structure on compact nilmanifolds $G / \Gamma$, where $\Gamma \subset G$ is a lattice, acting by left-multiplication on $G$. According to a theorem of Malcev [17] a simply connected nilpotent Lie group $G$ has a lattice if and only if its Lie algebra $\mathfrak{g}$ admits a basis with rational structure constants. This is the case in all examples that we consider.

In this paper we discuss the existence of bi-Lagrangian structures on symplectic nilpotent Lie algebras $\mathfrak{g}$ of dimension 2, 4 and 6. Existence of a bi-Lagrangian structure is shown by exhibiting a specific example of a symplectic form $\omega$ on $\mathfrak{g}$ and two complementary Lagrangian subalgebras $\mathcal{F}, \mathcal{G} \subset \mathfrak{g}$. Proving non-existence of a bi-Lagrangian structure (for any symplectic form on $\mathfrak{g}$ ) is more involved. The main result can be summarized as follows:

Theorem 1.4. (a) In dimension 2 there is a single nilpotent Lie algebra up to isomorphism. It admits a bi-Lagrangian structure.

(b) In dimension 4 there are three nilpotent Lie algebras up to isomorphism, each of which admits a symplectic form. Two of them admit a bi-Lagrangian structure and one of them does not (see Table 1 in the appendix).

(c) In dimension 6 there are 26 nilpotent Lie algebras up to isomorphism that admit a symplectic form. 16 of them admit a bi-Lagrangian structure and 10 of them do not (see Table 4).

Remark 1.5. The general existence question for a single Lagrangian foliation on nilpotent Lie algebras has been settled before by Baues and Cortés: According to Corollary 3.13. in [2] every symplectic form on a nilpotent Lie algebra admits a Lagrangian foliation.

It is an interesting observation, originally perhaps due to H. Hess [13], that every bi-Lagrangian structure $(\omega, \mathcal{F}, \mathcal{G})$ on a smooth, $2 n$-dimensional manifold $M$ defines an associated canonical affine connection $\nabla$ on $T M$, see Section 2 for the definition. It has the following properties: 
(a) $\nabla$ preserves both subbundles $T \mathcal{F}$ and $T \mathcal{G}$

(b) $\nabla$ is symplectic, i.e. $\omega$ is parallel with respect to $\nabla$

(c) $\nabla$ is torsion-free: $\nabla_{X} Y-\nabla_{Y} X=[X, Y]$ for all $X, Y \in \mathfrak{X}(M)$.

Remark 1.6. The canonical connection of a bi-Lagrangian structure is uniquely characterized by these three properties.

The canonical connection coincides with the Levi-Civita connection of a certain pseudo-Riemannian metric $g$ associated to the bi-Lagrangian structure: We denote the projections of a vector field $X$ onto $T \mathcal{F}$ and $T \mathcal{G}$ by $X_{F}$ and $X_{G}$ and define

$$
I: T M \longrightarrow T M, \quad X_{F}+X_{G} \longmapsto X_{F}-X_{G}
$$

and

$$
g: T M \times T M \longrightarrow \mathbb{R}, \quad(X, Y) \longmapsto \omega(I X, Y) .
$$

Then $I^{2}=\operatorname{Id}_{T M}$ and $g$ is a pseudo-Riemannian metric of neutral signature $(n, n)$ on the manifold $M^{2 n}$. The pair $(I, g)$ is called a para-Kähler structure on $M$. It turns out that the canonical connection of the bi-Lagrangian structure $(\omega, \mathcal{F}, \mathcal{G})$ is equal to the Levi-Civita connection of $g$; $\mathrm{cf}$. [8, 9].

We are interested especially in the curvature of the canonical connection. It is easy to see that for the standard bi-Lagrangian structure $\left(\omega_{0}, \mathcal{F}_{0}, \mathcal{G}_{0}\right)$ on $\mathbb{R}^{2 n}$, considered in Example 1.2, the curvature tensor $R$ vanishes identically. There is a Darboux-type converse to this statement: The curvature of the canonical connection of any bi-Lagrangian structure vanishes if and only if it is locally isomorphic to the standard structure $\left(\omega_{0}, \mathcal{F}_{0}, \mathcal{G}_{0}\right)$.

We calculate the curvature of the canonical connection for all our examples and show:

Theorem 1.7. $\quad$ (a) In dimension 2 and 4 the canonical connection is flat for all our examples of bi-Lagrangian structures.

(b) In dimension 6 the canonical connection is flat for 8 examples and non-flat for the remaining 8 examples of bi-Lagrangian structures. All our examples of bi-Lagrangian structures are Ricci-flat (see Table 5).

Examples of non-flat, Ricci-flat para-Kähler structures on nilpotent Lie algebras have been constructed before in [18] and [6]. The latter reference contains a general study of the Ricci curvature of para-Kähler structures (and, more generally, almost para-Hermitian structures, such as nearly para-Kähler structures).

An analysis of the (Ricci) curvature of related structures, such as tri-Lagrangian structures studied in [10, 7], will be left for future research.

Notation. All nilpotent Lie algebras that we consider are real. For a nilpotent Lie algebra $\mathfrak{g}$ we denote by $e_{1}, \ldots, e_{n}$ a basis and by $\alpha_{1}, \ldots, \alpha_{n}$ the dual basis. The differentials $d \alpha_{i}$ are related to the commutators $\left[e_{j}, e_{k}\right]$ by

$$
d \alpha_{i}\left(e_{j}, e_{k}\right)=-\alpha_{i}\left(\left[e_{j}, e_{k}\right]\right) .
$$

We set $\alpha_{i j}=\alpha_{i} \wedge \alpha_{j}$. A foliation (subalgebra) $\mathcal{F}$ in a nilpotent Lie algebra is specified by a basis $\left\{f_{1}, \ldots, f_{m}\right\}$. 
We follow the notation for Lie algebras in [3]: $A_{n}$ denotes an $n$-dimensional abelian Lie algebra and $L_{m}$ or $L_{m, k}$ an $m$-dimensional nilpotent Lie algebra.

In the calculation of the canonical connection $\nabla$ of a bi-Lagrangian structure only the non-vanishing components in the bases for the Lagrangian foliations are given. In the calculation of the curvature tensor $R$ all non-zero components up to the symmetry $R(X, Y) Z=-R(Y, X) Z$ are given.

\section{Preparations}

Suppose that $\mathfrak{g}$ is a 4 - or 6 -dimensional nilpotent Lie algebra and we want to prove that $\mathfrak{g}$ does not admit a bi-Lagrangian structure. We will frequently use the following standard lemma.

Lemma 2.1. Every subalgebra of a nilpotent Lie algebra is nilpotent. If $\mathfrak{h}$ is an $n$-dimensional nilpotent Lie algebra, then $\operatorname{dim}[\mathfrak{h}, \mathfrak{h}] \leq n-2$. In particular, every 2-dimensional nilpotent Lie algebra is abelian and every 3-dimensional nilpotent Lie algebra $\mathfrak{h}$ satisfies $[\mathfrak{h},[\mathfrak{h}, \mathfrak{h}]]=0$.

Lemma2.1 restricts the possible vector subspaces in $\mathfrak{g}$ that are subalgebras and hence define foliations. Together with the condition that the symplectic form $\omega$ is closed and non-degenerate this can be used to rule out the existence of a biLagrangian structure on $\mathfrak{g}$.

Suppose that $(\omega, \mathcal{F}, \mathcal{G})$ is a bi-Lagrangian structure on a smooth manifold $M$. We want to define the associated canonical connection: For vector fields $X, Y \in$ $\mathfrak{X}(M)$ let $D(X, Y) \in \mathfrak{X}(M)$ be the unique vector field defined by

$$
i_{D(X, Y)} \omega=L_{X} i_{Y} \omega
$$

Then we set

$$
\begin{array}{ll}
\nabla_{X} Y=D\left(X_{F}, Y\right)_{F}+\left[X_{G}, Y\right]_{F} & \forall Y \in \Gamma(T \mathcal{F}) \\
\nabla_{X} Y=D\left(X_{G}, Y\right)_{G}+\left[X_{F}, Y\right]_{G} & \forall Y \in \Gamma(T \mathcal{G}) .
\end{array}
$$

One can check that these expressions define connections on the vector bundles $T \mathcal{F}$, $T \mathcal{G}$. The direct sum defines an affine connection $\nabla$ on $T M$, called the canonical connection or Künneth connection.

The curvature $R$ of the canonical connection is defined in the standard way by

$$
R(X, Y) Z=\nabla_{X} \nabla_{Y} Z-\nabla_{Y} \nabla_{X} Z-\nabla_{[X, Y]} Z \text {. }
$$

The curvature tensor satisfies the Bianchi identity

$$
R(X, Y) Z+R(Y, Z) X+R(Z, X) Y=0 \quad \forall X, Y, Z \in T M .
$$

In addition, the tangent bundle $T M$ is flat along the leaves of both $\mathcal{F}$ and $\mathcal{G}$ :

$$
R(X, Y) Z=0 \quad \forall Z \in T M
$$

whenever $X, Y \in T \mathcal{F}$ or $X, Y \in T \mathcal{G}$. Together with the Bianchi identity we get the symmetry

$$
R(X, Y) Z=R(X, Z) Y \quad \forall X \in T M
$$

whenever $Y, Z \in T \mathcal{F}$ or $Y, Z \in T \mathcal{G}$. 
Finally, the Ricci curvature $\operatorname{Ric}(X, Y)$ of vectors $X, Y \in T M$ is defined as the trace of the map

$$
T M \longrightarrow T M, \quad Z \longmapsto R(Z, X) Y .
$$

\section{TWO- AND FOUR-DIMENSIONAL NILPOTENT LIE ALGEBRAS}

The case of dimension 2 is trivial: there is a single nilpotent 2-dimensional Lie algebra, the abelian Lie algebra $A_{2}$. A bi-Lagrangian structure is given by the symplectic form $\omega=\alpha_{12}$ and the Lagrangian foliations $\mathcal{F}=\left\{e_{1}\right\}, \mathcal{G}=\left\{e_{2}\right\}$. The canonical connection $\nabla$ is trivial in the basis $e_{1}, e_{2}$ and its curvature $R$ vanishes.

We now consider the 4-dimensional case, see Table 1 in the appendix. There are three nilpotent Lie algebras of dimension $4, A_{4}, L_{3} \oplus A_{1}$ and $L_{4}$, each of which is symplectic. The first two admit a bi-Lagrangian structure, while the third one does not. We prove non-existence for any symplectic form in the case of $L_{4}$ in the following subsection.

\subsection{Non-existence of a bi-Lagrangian structure on $L_{4}$.}

Lemma 3.1. Suppose that $L_{4}$ has two complementary 2-dimensional subalgebras $\mathcal{F}, \mathcal{G}$. Then one of the two subalgebras, say $\mathcal{F}$, has a basis of the form

$$
e_{1}+a_{2} e_{2}+a_{3} e_{3}, \quad e_{4} \text {. }
$$

Proof. It is clear that one of the subalgebras has a basis vector of the form

$$
f_{1}=e_{1}+\sum_{i=2}^{4} a_{i} e_{i}
$$

Any other basis vector of the same subalgebra can be assumed to be of the form

$$
f_{2}=\sum_{j=2}^{4} b_{j} e_{j}
$$

We get

$$
\left[f_{1}, f_{2}\right]=-b_{2} e_{3}-b_{3} e_{4} .
$$

Lemma2.1 implies that $b_{2}=b_{3}=0$, hence the claim.

Proposition 3.2. The Lie algebra $L_{4}$ does not admit a bi-Lagrangian structure.

Proof. Any closed 2-form on $L_{4}$ is of the form

$$
\omega=\omega_{12} \alpha_{12}+\omega_{13} \alpha_{13}+\omega_{23} \alpha_{23}+\omega_{14} \alpha_{14} .
$$

If the form $\omega$ is symplectic, then $\omega_{14} \neq 0$. It follows that the subalgebra $\mathcal{F}$ in Lemma 3.1 cannot be Lagrangian.

3.2. Calculation of the curvature of bi-Lagrangian structures in Table 1, We calculate the canonical connection and the curvature of the bi-Lagrangian structure for $A_{4}$ and $L_{3} \oplus A_{1}$. It turns out that both examples are flat.

3.2.1. $A_{4}$. A simple calculation shows that the canonical connection $\nabla$ is trivial in the basis $e_{1}, e_{2}, e_{3}, e_{4}$ and $R=0$.

3.2.2. $L_{3} \oplus A_{1}$.

$$
\begin{gathered}
\nabla_{e_{1}} e_{1}=-e_{3}, \quad \nabla_{e_{1}} e_{2}=-e_{4} . \\
R=0 .
\end{gathered}
$$




\section{SIX-DIMENSIONAL NILPOTENT LIE ALGEBRAS}

We now consider the 6 -dimensional case. The symplectic nilpotent Lie algebras of dimension 6 have been determined (independently) by [19], [16] and [3]. The latter two references contain explicit symplectic forms. See Table 2 for a comparison of notation in these three references, Table 3 for the structure constants and Table 4 for symplectic forms and bi-Lagrangian structures.

Remark 4.1. The lists in [3] and [16] for symplectic forms on 6-dimensional nilpotent Lie algebras contain several errors:

- The 2-forms in [3, Table 3] for the following Lie algebras are not symplectic:

$$
L_{6,1}, L_{6,12}, L_{6,15}, L_{6,16}, L_{6,17}^{-} .
$$

- The following 2-forms in [16, Section 3] are not symplectic:

$$
\text { 16. } \omega_{2} \text { and 23. } \omega_{3} \text {. }
$$

Because of this error in [16] we do not use the classification of symplectic forms (up to automorphisms of the Lie algebras) that is stated in this reference.

There are in total 26 nilpotent 6 -dimensional Lie algebras (up to isomorphism) which admit a symplectic form, 16 of which admit a bi-Lagrangian structure and 10 of which do not admit such a structure. If a bi-Lagrangian structure exists, an example is given in Table 4. We now prove non-existence (for any symplectic form) in the remaining cases.

4.1. Non-existence of bi-Lagrangian structures. We will repeatedly use the following lemma.

Lemma 4.2. Let $\mathfrak{g}$ be a 6-dimensional Lie algebra with basis $e_{1}, \ldots, e_{6}$.

(a) If a 3-dimensional vector subspace $\mathcal{F} \subset \mathfrak{g}$ has a basis vector with a nonzero $e_{1}$-component, then a basis of $\mathcal{F}$ is of the form

$$
f_{1}=e_{1}+\sum_{i=2}^{6} a_{i} e_{i}, \quad f_{2}=\sum_{j=2}^{6} b_{j} e_{j}, \quad f_{3}=\sum_{k=2}^{6} c_{k} e_{k},
$$

with $a_{i}, b_{j}, c_{k} \in \mathbb{R}$.

(b) If $\mathfrak{g}=\mathcal{F} \oplus \mathcal{G}$ is a sum of two 3-dimensional vector subspaces, then at least one of $\mathcal{F}, \mathcal{G}$ has a basis as in $(a)$.

4.1.1. $L_{4} \oplus A_{2}$.

Lemma 4.3. A 2-form $\omega$ on $L_{4} \oplus A_{2}$ is closed if and only if it is of the form

$$
\begin{aligned}
\omega= & \omega_{12} \alpha_{12}+\omega_{13} \alpha_{13}+\omega_{23} \alpha_{23}+\omega_{14} \alpha_{14}+\omega_{24} \alpha_{24}+\omega_{34} \alpha_{34} \\
& +\omega_{15} \alpha_{15}+\omega_{25} \alpha_{25}+\omega_{16} \alpha_{16} .
\end{aligned}
$$

The form is symplectic if and only if $\omega_{16} \omega_{25} \omega_{34} \neq 0$.

Lemma 4.4. Suppose that $L_{4} \oplus A_{2}$ has two complementary 3-dimensional subalgebras $\mathcal{F}, \mathcal{G}$. Then one of the two subalgebras, say $\mathcal{F}$, has a basis of either one of the following forms: 

(a) $e_{1}+a_{2} e_{2}+a_{3} e_{3}+a_{4} e_{4}, \quad e_{5}+b_{3} e_{3}+b_{4} e_{4}, \quad e_{6}$
(b) $e_{1}+a_{2} e_{2}+a_{5} e_{5}+a_{6} e_{6}, \quad e_{3}+b_{6} e_{6}, \quad e_{4}+c_{6} e_{6}$
(c) $e_{1}+a_{2} e_{2}+a_{4} e_{4}+a_{5} e_{5}, \quad e_{3}+b_{4} e_{4}, \quad e_{6}$
(d) $e_{1}+a_{2} e_{2}+a_{3} e_{3}+a_{5} e_{5}, \quad e_{4}, \quad e_{6}$.

Proof. Suppose that $\mathcal{F}$ has basis vectors $f_{1}, f_{2}$ as in Lemma 4.2 (a). We get

$$
\left[f_{1}, f_{2}\right]=-\left(b_{2} e_{5}+b_{5} e_{6}\right)
$$

and $\left[f_{1},\left[f_{1}, f_{2}\right]\right]=b_{2} e_{6}$. Lemma2.1 implies that $b_{2}=0$. A third basis vector is of the form

$$
f_{3}=c_{3} e_{3}+c_{4} e_{4}+c_{5} e_{5}+c_{6} e_{6}
$$

There are two cases:

- $b_{5} \neq 0$ and $c_{5}=0$. Then equation (4.1) shows that $e_{6} \in \mathcal{F}$. This results in a basis of the first form.

- $b_{5}=0$ and $c_{5}=0$. Then the remaining two basis vectors are of the form

$$
f_{2}=b_{3} e_{3}+b_{4} e_{4}+b_{6} e_{6}, \quad f_{3}=c_{3} e_{3}+c_{4} e_{4}+c_{6} e_{6} .
$$

If $b_{3} \neq 0$ and $c_{3}=0$ we get the bases in (b) and (c), depending on whether $c_{4} \neq 0$ or $c_{4}=0$. If $b_{3}=0$ and $c_{3}=0$ we get the basis in (d).

Proposition 4.5. The Lie algebra $L_{4} \oplus A_{2}$ does not admit a bi-Lagrangian structure.

Proof. For the symplectic form as in Lemma 4.3 the subalgebra $\mathcal{F}$ in Lemma 4.4 cannot be Lagrangian.

4.1.2. $L_{6,13}$.

Lemma 4.6. Every closed 2-form on $L_{6,13}$ is of the form

$$
\begin{aligned}
\omega= & \omega_{12} \alpha_{12}+\omega_{13} \alpha_{13}+\omega_{23} \alpha_{23}+\omega_{14} \alpha_{14}+\omega_{24} \alpha_{24}-\omega_{16} \alpha_{34} \\
& +\omega_{15} \alpha_{15}-\omega_{26} \alpha_{45}+\omega_{16} \alpha_{16}+\omega_{26} \alpha_{26} .
\end{aligned}
$$

If $\omega$ is symplectic, then $\omega_{26}$ has to be nonzero.

Lemma 4.7. Let $\mathcal{F}$ be a 3-dimensional subalgebra of $L_{6,13}$ with a basis vector having a non-zero $e_{1}$-component. Then $\mathcal{F}$ has a basis of either one of the following two forms:

(a) $e_{1}+a_{2} e_{2}+a_{4} e_{4}+a_{5} e_{5}, \quad e_{3}+b_{5} e_{5}, \quad e_{6}$

(b) $e_{1}+a_{2} e_{2}+a_{3} e_{3}+a_{4} e_{4}, \quad e_{5}, \quad e_{6}$.

Proof. Suppose that $\mathcal{F}$ has basis vectors $f_{1}, f_{2}$ as in Lemma4.2 (a). We get

$$
\left[f_{1}, f_{2}\right]=-\left(b_{2} e_{4}+b_{4} e_{5}+\left(b_{5}+a_{2} b_{3}-a_{3} b_{2}\right) e_{6}\right)
$$

and $\left[f_{1},\left[f_{1}, f_{2}\right]\right]=b_{2} e_{5}+b_{4} e_{6}$. Lemma 2.1 implies that $b_{2}=b_{4}=0$. There are two cases:

- $b_{3}=0$. Then either $b_{5}=0$, hence $b_{6} \neq 0$ and $e_{6} \in \mathcal{F}$. This results in a basis of type (a) or (b). Or $b_{5} \neq 0$ and equation (4.2) shows that $e_{6} \in \mathcal{F}$. This results in a basis of type (b). 
- $b_{3} \neq 0$. A third basis vector of $\mathcal{F}$ is then of the form

$$
f_{3}=c_{5} e_{5}+c_{6} e_{6} \text {. }
$$

If $c_{5}=0$, then $c_{6} \neq 0$ and $e_{6} \in \mathcal{F}$. This results in a basis of type (a). If $c_{5} \neq 0$, then the equation $\left[f_{1}, f_{3}\right]=-c_{5} e_{6}$ again shows that $e_{6} \in \mathcal{F}$. This case cannot occur, because then $\mathcal{F}$ had to be at least 4-dimensional.

Proposition 4.8. The Lie algebra $L_{6,13}$ does not admit a bi-Lagrangian structure.

Proof. Suppose that $\mathcal{F}, \mathcal{G}$ is a bi-Lagrangian structure. Considering the bases in Lemma 4.7 it follows that exactly one of $\mathcal{F}, \mathcal{G}$ must have a basis with a non-zero $e_{1}$-component, say $\mathcal{F}$. Otherwise $\mathcal{F}$ and $\mathcal{G}$ cannot be complementary.

In the first case $\mathcal{F}$ has a basis of the form

$$
e_{1}+a_{2} e_{2}+a_{4} e_{4}+a_{5} e_{5}, \quad e_{3}+b_{5} e_{5}, \quad e_{6} .
$$

It follows that $\mathcal{G}$ has a basis of the form

$$
\begin{aligned}
& g_{1}=e_{2}+x_{3} e_{3}+x_{5} e_{5}+x_{6} e_{6} \\
& g_{2}=e_{4}+y_{3} e_{3}+x_{5} e_{5}+y_{6} e_{6} \\
& g_{3}=z_{3} e_{3}+z_{5} e_{5}+z_{6} e_{6} .
\end{aligned}
$$

We have $\left[g_{1}, g_{2}\right]=-y_{3} e_{6}$. This implies that $y_{3}=0$, since otherwise $\left[g_{1}, g_{2}\right] \in \mathcal{F}$. Similarly $\left[g_{1}, g_{3}\right]=-z_{3} e_{6}$, hence $z_{3}=0$. Then also $z_{5} \neq 0$, since otherwise $g_{3}$ is a multiple of $e_{6} \in \mathcal{F}$.

The basis is now

$$
g_{1}=e_{2}+x_{3} e_{3}+x_{6} e_{6}, \quad g_{2}=e_{4}+y_{6} e_{6}, \quad g_{3}=e_{5}+z_{6} e_{6} .
$$

Considering the symplectic form $\omega$ as in Lemma 4.6 and the fact that $\omega_{26} \neq 0$, it follows that $\omega\left(g_{2}, g_{3}\right) \neq 0$, hence $\mathcal{G}$ cannot be Lagrangian. This is a contradiction.

In the second case $\mathcal{F}$ has a basis of the form

$$
e_{1}+a_{2} e_{2}+a_{3} e_{3}+a_{4} e_{4}, \quad e_{5}, \quad e_{6} .
$$

It follows that $\mathcal{G}$ has a basis of the form

$$
g_{1}=e_{2}+x_{5} e_{5}+x_{6} e_{6}, \quad g_{2}=e_{3}+y_{5} e_{5}+y_{6} e_{6}, \quad g_{3}=e_{4}+z_{5} e_{5}+z_{6} e_{6} .
$$

We have $\left[g_{1}, g_{2}\right]=-e_{6} \in \mathcal{F}$. This is a contradiction.

\subsection{3. $L_{5,6} \oplus A_{1}$.}

Lemma 4.9. Let $\mathcal{F}$ be a 3 -dimensional subalgebra of $L_{5,6} \oplus A_{1}$ with a basis vector having a non-zero $e_{1}$-component. Then $\mathcal{F}$ has a basis of either one of the following two forms:
(a) $e_{1}+a_{2} e_{2}+a_{3} e_{3}+a_{4} e_{4}, \quad e_{5}+b_{3} e_{3}, \quad e_{6}$
(b) $e_{1}+a_{2} e_{2}+a_{4} e_{4}+a_{5} e_{5}, \quad e_{3}, \quad e_{6}$ 
Proof. Suppose that $\mathcal{F}$ has basis vectors $f_{1}, f_{2}$ as in Lemma 4.2 (a). We get

$$
\begin{aligned}
{\left[f_{1}, f_{2}\right] } & =-\left(b_{2} e_{4}+b_{4} e_{5}+\left(b_{5}+a_{2} b_{4}-a_{4} b_{2}\right) e_{6}\right) \\
{\left[f_{1},\left[f_{1}, f_{2}\right]\right] } & =b_{2} e_{5}+b_{4} e_{6}+a_{2} b_{2} e_{6} .
\end{aligned}
$$

Lemma2.1 implies that $b_{2}=b_{4}=0$. A third basis vector is of the form

$$
f_{3}=c_{3} e_{3}+c_{5} e_{5}+c_{6} e_{6} .
$$

There are two cases:

(a) $b_{5} \neq 0$ and $c_{5}=0$. Then equation (4.3) implies that $e_{6} \in \mathcal{F}$. This results in a basis of type (a).

(b) $b_{5}=0$ and $c_{5}=0$. This results in a basis of type (b).

Proposition 4.10. The Lie algebra $L_{5,6} \oplus A_{1}$ does not have two complementary 3-dimensional subalgebras $\mathcal{F}, \mathcal{G}$. In particular, $L_{5,6} \oplus A_{1}$ does not admit a biLagrangian structure.

Proof. Suppose that $\mathcal{F}, \mathcal{G}$ are two complementary 3-dimensional subalgebras of $L_{5,6} \oplus A_{1}$. Considering the bases in Lemma 4.9. it follows that exactly one of $\mathcal{F}, \mathcal{G}$ must have a basis with a non-zero $e_{1}$-component, say $\mathcal{F}$. Otherwise $\mathcal{F}$ and $\mathcal{G}$ cannot be complementary.

In the first case $\mathcal{F}$ has a basis of the form

$$
e_{1}+a_{2} e_{2}+a_{3} e_{3}+a_{4} e_{4}, \quad e_{5}+b_{3} e_{3}, \quad e_{6} .
$$

It follows that $\mathcal{G}$ has a basis of the form

$$
\begin{aligned}
& g_{1}=e_{2}+x_{3} e_{3}+x_{5} e_{5}+x_{6} e_{6} \\
& g_{2}=z_{3} e_{3}+y_{5} e_{5}+y_{6} e_{6} \\
& g_{3}=e_{4}+x_{3} e_{3}+z_{5} e_{5}+z_{6} e_{6} .
\end{aligned}
$$

Then $\left[g_{1}, g_{3}\right]=-e_{6} \in \mathcal{F}$. This is a contradiction.

In the second case $\mathcal{F}$ has a basis of the form

$$
e_{1}+a_{2} e_{2}+a_{4} e_{4}+a_{5} e_{5}, \quad e_{3}, \quad e_{6} .
$$

It follows that $\mathcal{G}$ has a basis of the form

$$
g_{1}=e_{2}+x_{3} e_{3}+x_{6} e_{6}, \quad g_{2}=e_{4}+y_{3} e_{3}+y_{6} e_{6}, \quad g_{3}=e_{5}+z_{3} e_{3}+z_{6} e_{6} .
$$

Then $\left[g_{1}, g_{2}\right]=-e_{6} \in \mathcal{F}$. This is a contradiction.

4.1.4. $L_{6,14}$.

Lemma 4.11. Every closed 2-form on $L_{6,14}$ is of the form

$$
\begin{aligned}
\omega= & \omega_{12} \alpha_{12}+\omega_{13} \alpha_{13}+\omega_{23} \alpha_{23}+\omega_{14} \alpha_{14}+\omega_{24} \alpha_{24}-\omega_{16} \alpha_{34} \\
& +\omega_{15} \alpha_{15}+\omega_{16} \alpha_{25}-\omega_{26} \alpha_{45}+\omega_{16} \alpha_{16}+\omega_{26} \alpha_{26} .
\end{aligned}
$$

If $\omega$ is symplectic, then at least one of $\omega_{16}, \omega_{26}$ has to be non-zero. 
Lemma 4.12. Let $\mathcal{F}$ be a 3 -dimensional subalgebra of $L_{6,14}$ with a basis vector having a non-zero $e_{1}$-component. Then $\mathcal{F}$ has a basis of either one of the following two forms:

(a) $e_{1}+a_{2} e_{2}+a_{4} e_{4}+a_{5} e_{5}, \quad e_{3}+b_{5} e_{5}, \quad e_{6}$

(b) $e_{1}+a_{2} e_{2}+a_{3} e_{3}+a_{4} e_{4}, \quad e_{5}, \quad e_{6}$.

If $\mathcal{F}$ is Lagrangian, then $\omega_{26} \neq 0$ for the symplectic form in Lemma 4.11

Proof. Suppose that $\mathcal{F}$ has basis vectors $f_{1}, f_{2}$ as in Lemma4.2 (a). We get

$$
\begin{aligned}
{\left[f_{1}, f_{2}\right] } & =-\left(b_{2} e_{4}+b_{4} e_{5}+\left(b_{5}+a_{2} b_{3}-a_{3} b_{2}+a_{2} b_{4}-a_{4} b_{2}\right) e_{6}\right) \\
{\left[f_{1},\left[f_{1}, f_{2}\right]\right] } & =b_{2} e_{5}+b_{4} e_{6}+a_{2} b_{2} e_{6} .
\end{aligned}
$$

Lemma 2.1 implies that $b_{2}=b_{4}=0$. There are two cases:

(a) $b_{3} \neq 0$. Then a third basis vector of $\mathcal{F}$ is of the form

$$
f_{3}=c_{5} e_{5}+c_{6} e_{6} .
$$

If $c_{5}=0$, then $c_{6} \neq 0$ and $e_{6} \in \mathcal{F}$. This results in a basis of type (a). If $c_{5} \neq 0$, then the equation $\left[f_{1}, f_{3}\right]=-c_{5} e_{6}$ implies again that $e_{6} \in \mathcal{F}$. This case cannot occur, because then $\mathcal{F}$ has to be at least 4-dimensional.

(b) $b_{3}=0$. If $b_{5}=0$, then $b_{6} \neq 0$, hence $e_{6} \in \mathcal{F}$. This results either in a basis of type (a) or (b). If $b_{5} \neq 0$, then equation (4.4) shows that $e_{6} \in \mathcal{F}$. This results in a basis of type (b).

Proposition 4.13. The Lie algebra $L_{6,14}$ does not have two complementary 3dimensional subalgebras $\mathcal{F}, \mathcal{G}$. In particular, $L_{6,14}$ does not admit a bi-Lagrangian structure.

Proof. Suppose that $L_{6,14}$ has two complementary 3-dimensional subalgebras $\mathcal{F}, \mathcal{G}$. Considering the bases in Lemma 4.12, it follows that exactly one of $\mathcal{F}, \mathcal{G}$ must have a basis with a non-zero $e_{1}$-component, say $\mathcal{F}$. Otherwise $\mathcal{F}$ and $\mathcal{G}$ cannot be complementary.

In the first case $\mathcal{F}$ has a basis of the form

$$
e_{1}+a_{2} e_{2}+a_{4} e_{4}+a_{5} e_{5}, \quad e_{3}+b_{5} e_{5}, \quad e_{6} .
$$

It follows that $\mathcal{G}$ has a basis of the form

$$
\begin{aligned}
& g_{1}=e_{2}+x_{3} e_{3}+x_{5} e_{5}+x_{6} e_{6} \\
& g_{2}=e_{4}+y_{3} e_{3}+y_{5} e_{5}+y_{6} e_{6} \\
& g_{3}=z_{3} e_{3}+z_{5} e_{5}+z_{6} e_{6} .
\end{aligned}
$$

We have $\left[g_{1}, g_{2}\right]=-e_{6}-y_{3} e_{6}$. Hence $y_{3}=-1$, otherwise $\left[g_{1}, g_{2}\right]$ is a multiple of $e_{6} \in \mathcal{F}$. Similarly, $\left[g_{1}, g_{3}\right]=-z_{3} e_{6} \in \mathcal{F}$, hence $z_{3}=0$. Then also $z_{5} \neq 0$, since otherwise $g_{3}$ is a multiple of $e_{6} \in \mathcal{F}$.

The basis is now of the form

$$
g_{1}=e_{2}+x_{3} e_{3}+x_{6} e_{6}, \quad g_{2}=e_{4}-e_{3}+y_{6} e_{6}, \quad g_{3}=e_{5}+z_{6} e_{6} .
$$


Considering the symplectic form $\omega$ as in Lemma 4.11 and the fact that $\omega_{26} \neq$ 0 according to Lemma 4.12, it follows that $\omega\left(g_{2}, g_{3}\right) \neq 0$, hence $\mathcal{G}$ cannot be Lagrangian. This is a contradiction.

In the second case $\mathcal{F}$ has a basis of the form

$$
e_{1}+a_{2} e_{2}+a_{3} e_{3}+a_{4} e_{4}, \quad e_{5}, \quad e_{6} .
$$

It follows that $\mathcal{G}$ has a basis of the form

$$
g_{1}=e_{2}+x_{5} e_{5}+x_{6} e_{6}, \quad g_{2}=e_{3}+y_{5} e_{5}+y_{6} e_{6}, \quad g_{3}=e_{4}+z_{5} e_{5}+z_{6} e_{6} .
$$

Then $\left[g_{1}, g_{2}\right]=-e_{6} \in \mathcal{F}$. This is a contradiction.

4.1.5. $L_{6,15}$.

Lemma 4.14. A 2-form $\omega$ on $L_{6,15}$ is closed if and only if it is of the form

$$
\begin{aligned}
\omega= & \omega_{12} \alpha_{12}+\omega_{13} \alpha_{13}+\omega_{23} \alpha_{23}+\omega_{14} \alpha_{14}+\omega_{24} \alpha_{24}-\omega_{15} \alpha_{34} \\
& +\omega_{15} \alpha_{15}-\omega_{16} \alpha_{35}+\omega_{16} \alpha_{16} .
\end{aligned}
$$

If the form $\omega$ is symplectic, then $\omega_{16} \neq 0$.

Lemma 4.15. Suppose that $L_{6,15}$ has two complementary 3-dimensional subalgebras $\mathcal{F}, \mathcal{G}$. Then one of the two subalgebras, say $\mathcal{F}$, has a basis that contains two vectors of the following form:

$$
e_{1}+\sum_{i=2}^{5} a_{i} e_{i}, \quad e_{6} .
$$

Proof. Suppose that $\mathcal{F}$ has basis vectors $f_{1}, f_{2}$ as in Lemma 4.2 (a). We get

$$
\begin{aligned}
{\left[f_{1}, f_{2}\right] } & =-\left(b_{2} e_{4}+\left(b_{4}+a_{2} b_{3}-a_{3} b_{2}\right) e_{5}+\left(b_{5}-a_{3} b_{4}+a_{4} b_{3}\right) e_{6}\right) \\
{\left[f_{1},\left[f_{1}, f_{2}\right]\right] } & =b_{2} e_{5}+\left(b_{4}+a_{2} b_{3}-2 a_{3} b_{2}\right) e_{6} .
\end{aligned}
$$

Lemma 2.1 implies that $b_{2}=0$ and $b_{4}+a_{2} b_{3}-2 a_{3} b_{2}=0$. There are two cases:

(a) $b_{5}-a_{3} b_{4}+a_{4} b_{3} \neq 0$. Then equation (4.5) shows that $e_{6} \in \mathcal{F}$.

(b) $b_{5}-a_{3} b_{4}+a_{4} b_{3}=0$. A third basis vector of $\mathcal{F}$ is then of the form

$$
f_{3}=c_{3} e_{3}+c_{4} e_{4}+c_{5} e_{5}+c_{6} e_{6}
$$

with $c_{4}+a_{2} c_{3}-2 a_{3} c_{2}=0$. There are then two subcases:

- $b_{3}=0$ and $c_{3}=0$. Then also $b_{4}=b_{5}=0$, hence only $b_{6} \neq 0$ and $e_{6} \in \mathcal{F}$.

- $b_{3} \neq 0$ and $c_{3}=0$. Then also $c_{4}=0$. If $c_{5} \neq 0$, then the equation $\left[f_{1}, f_{3}\right]=-c_{5} e_{6}$ shows that $e_{6} \in \mathcal{F}$. This case cannot occur, because then $\mathcal{F}$ had to be at least 4 -dimensional. If $c_{5}=0$, then only $c_{6} \neq 0$ and again $e_{6} \in \mathcal{F}$.

Proposition 4.16. The Lie algebra $L_{6,15}$ does not admit a bi-Lagrangian structure.

Proof. Considering a symplectic form as in Lemma 4.14 it follows that the subalgebra $\mathcal{F}$ in Lemma 4.15 cannot be Lagrangian. 
4.1.6. $L_{6,17}^{+}$.

Lemma 4.17. Let $\mathcal{F}$ be a 3-dimensional subalgebra of $L_{6,17}^{+}$with a basis vector having a non-zero $e_{1}$-component. Then $\mathcal{F}$ has a basis of either one of the following two forms:

(a) $e_{1}+a_{2} e_{2}+a_{3} e_{3}+a_{5} e_{5}, \quad e_{4}, \quad e_{6}$

(b) $e_{1}+a_{2} e_{2}+a_{3} e_{3}+a_{4} e_{4}, \quad e_{5}+b_{4} e_{4}, \quad e_{6}$

Proof. Suppose that $\mathcal{F}$ has basis vectors $f_{1}, f_{2}$ as in Lemma 4.2 (a). We get

$$
\left[f_{1}, f_{2}\right]=-\left(b_{2} e_{3}+b_{3} e_{4}+\left(a_{2} b_{3}-a_{3} b_{2}\right) e_{5}+\left(b_{4}+a_{2} b_{5}-a_{5} b_{2}\right) e_{6}\right)
$$

$\left[f_{1},\left[f_{1}, f_{2}\right]\right]=b_{2} e_{4}+a_{2} b_{2} e_{5}+\left(b_{3}\left(1+a_{2}^{2}\right)-a_{2} a_{3} b_{2}\right) e_{6}$.

Lemma2.1 implies that $b_{2}=b_{3}=0$. There are two cases:

(a) $b_{5}=0$. If also $b_{4}=0$, then only $b_{6} \neq 0$ and $e_{6} \in \mathcal{F}$. This results in a basis of type (a) or (b). If $b_{4} \neq 0$, then equation (4.6) shows that $e_{6} \in \mathcal{F}$. This results in a basis of type (a).

(b) $b_{5} \neq 0$. A third basis vector of $\mathcal{F}$ is then of the form

$$
f_{3}=c_{4} e_{4}+c_{6} e_{6} \text {. }
$$

If $c_{4}=0$, then $c_{6} \neq 0$ and $e_{6} \in \mathcal{F}$. This results in a basis of type (b). If $c_{4} \neq 0$, then the equation $\left[f_{1}, f_{3}\right]=-c_{4} e_{6}$ again implies that $e_{6} \in \mathcal{F}$. This case cannot occur, because then $\mathcal{F}$ had to be at least 4-dimensional.

Proposition 4.18. The Lie algebra $L_{6,17}^{+}$does not have two complementary 3dimensional subalgebras $\mathcal{F}, \mathcal{G}$. In particular, $L_{6,17}^{+}$does not admit a bi-Lagrangian structure.

Proof. Suppose that $L_{6,17}^{+}$has two complementary 3-dimensional subalgebras $\mathcal{F}, \mathcal{G}$. Considering the bases in Lemma 4.17, it follows that exactly one of $\mathcal{F}, \mathcal{G}$ must have a basis with a non-zero $e_{1}$-component, say $\mathcal{F}$. Otherwise $\mathcal{F}$ and $\mathcal{G}$ cannot be complementary.

In the first case $\mathcal{F}$ has a basis of the form

$$
e_{1}+a_{2} e_{2}+a_{3} e_{3}+a_{5} e_{5}, \quad e_{4}, \quad e_{6} .
$$

It follows that $\mathcal{G}$ has a basis of the form

$$
g_{1}=e_{2}+x_{4} e_{4}+x_{6} e_{6}, \quad g_{2}=e_{3}+y_{4} e_{4}+y_{6} e_{6}, \quad g_{3}=e_{5}+z_{4} e_{4}+z_{6} e_{6} .
$$

We get $\left[g_{1},\left[g_{1}, g_{2}\right]\right]=e_{6} \in \mathcal{F}$. This is a contradiction.

In the second case $\mathcal{F}$ has a basis of the form

$$
e_{1}+a_{2} e_{2}+a_{3} e_{3}+a_{4} e_{4}, \quad e_{5}+b_{4} e_{4}, \quad e_{6} .
$$

It follows that $\mathcal{G}$ has a basis of the form

$$
\begin{aligned}
& g_{1}=e_{2}+x_{4} e_{4}+x_{5} e_{5}+x_{6} e_{6} \\
& g_{2}=e_{3}+y_{4} e_{4}+y_{5} e_{5}+y_{6} e_{6} \\
& g_{3}=z_{4} e_{4}+z_{5} e_{5}+z_{6} e_{6} .
\end{aligned}
$$


We get $\left[g_{1},\left[g_{1}, g_{2}\right]\right]=e_{6} \in \mathcal{F}$. This is again a contradiction.

4.1.7. $L_{6,17}^{-}$.

Lemma 4.19. Every closed 2-form on $L_{6,17}^{-}$is of the form

$$
\begin{aligned}
\omega= & \omega_{12} \alpha_{12}+\omega_{13} \alpha_{13}+\omega_{23} \alpha_{23}+\omega_{14} \alpha_{14}+\omega_{15} \alpha_{24}-\omega_{26} \alpha_{34} \\
& +\omega_{15} \alpha_{15}+\omega_{25} \alpha_{25}-\omega_{16} \alpha_{35}+\omega_{16} \alpha_{16}+\omega_{26} \alpha_{26} .
\end{aligned}
$$

The form $\omega$ is symplectic if and only if $\left(\omega_{16}^{2}+\omega_{26}^{2}\right) \omega_{15}-\omega_{16} \omega_{26}\left(\omega_{25}+\omega_{14}\right) \neq 0$.

Lemma 4.20. Let $\mathcal{F}$ be a 3-dimensional subalgebra of $L_{6,17}^{-}$with a basis vector having a non-zero $e_{1}$-component. Then $\mathcal{F}$ has a basis of either one of the following four forms:

(a) $e_{1}+a_{2} e_{2}+a_{3} e_{3}+a_{5} e_{5}, \quad e_{4}, \quad e_{6}$

(b) $e_{1}+a_{2} e_{2}+a_{3} e_{3}+a_{4} e_{4}, \quad e_{5}+b_{4} e_{4}, \quad e_{6}$

(c) $e_{1}+e_{2}+a_{5} e_{5}+a_{6} e_{6}, \quad e_{3}+b_{5} e_{5}+b_{6} e_{6}, \quad e_{4}+e_{5}-b_{5} e_{6}$

(d) $e_{1}-e_{2}+a_{5}^{\prime} e_{5}+a_{6}^{\prime} e_{6}, \quad e_{3}+b_{5}^{\prime} e_{5}+b_{6}^{\prime} e_{6}, \quad e_{4}-e_{5}+b_{5}^{\prime} e_{6}$

Subalgebras with bases in (c) and (d) cannot be Lagrangian for any symplectic form on $L_{6,17}^{-}$.

Proof. Suppose that $\mathcal{F}$ has basis vectors $f_{1}, f_{2}$ as in Lemma4.2 (a). We get

$$
\begin{aligned}
{\left[f_{1}, f_{2}\right] } & =-\left(b_{2} e_{3}+b_{3} e_{4}+\left(a_{2} b_{3}-a_{3} b_{2}\right) e_{5}+\left(b_{4}-a_{2} b_{5}+a_{5} b_{2}\right) e_{6}\right) \\
{\left[f_{1},\left[f_{1}, f_{2}\right]\right] } & =b_{2} e_{4}+a_{2} b_{2} e_{5}+\left(b_{3}\left(1-a_{2}^{2}\right)+a_{2} a_{3} b_{2}\right) e_{6} .
\end{aligned}
$$

Lemma 2.1 implies that $b_{2}=0$ and $b_{3}\left(1-a_{2}^{2}\right)=0$. A third basis vector of $\mathcal{F}$ is then of the form

$$
f_{3}=c_{3} e_{3}+c_{4} e_{4}+c_{5} e_{5}+c_{6} e_{6}
$$

with $c_{3}\left(1-a_{2}^{2}\right)=0$. There are two cases:

(a) $b_{3}=0$ and $c_{3}=0$. There are two subcases:

- $b_{5}=0$. If also $b_{4}=0$, then only $b_{6} \neq 0$ and $e_{6} \in \mathcal{F}$. This results in a basis of type (a) or (b). If $b_{4} \neq 0$, then equation (4.7) shows that $e_{6} \in \mathcal{F}$. This results in a basis of type (a).

- $b_{5} \neq 0$. The third basis vector of $\mathcal{F}$ is then of the form

$$
f_{3}=c_{4} e_{4}+c_{6} e_{6} \text {. }
$$

If $c_{4}=0$, then $c_{6} \neq 0$ and $e_{6} \in \mathcal{F}$. This results in a basis of type (b). If $c_{4} \neq 0$, then the equation $\left[f_{1}, f_{3}\right]=-c_{4} e_{6}$ again implies that $e_{6} \in \mathcal{F}$. This case cannot occur, because then $\mathcal{F}$ had to be at least 4-dimensional.

(b) $b_{3} \neq 0$ and $c_{3}=0$. Then $a_{2}= \pm 1$. According to equation (4.7)

$$
\left[f_{1}, f_{2}\right]=-b_{3} e_{4}-a_{2} b_{3} e_{5}-\left(b_{4}-a_{2} b_{5}\right) e_{6} .
$$

It follows that we can assume that the third basis vector $f_{3}$ is $\left[f_{1}, f_{2}\right]$ up to a non-zero multiple, hence

$$
f_{2}=e_{3}+b_{5} e_{5}+b_{6} e_{6}, \quad f_{3}=e_{4} \pm e_{5} \mp b_{5} e_{6} .
$$

This results in a basis of type (c) or (d). 
Suppose that the basis in (c) spans a Lagrangian subspace for a symplectic form as in Lemma4.19. Then the following pairings of the first and third and second and third basis vector have to vanish:

$$
\omega_{14}+\omega_{15}-b_{5} \omega_{16}+\omega_{15}+\omega_{25}-b_{5} \omega_{26}=0, \quad-\omega_{26}-\omega_{16}=0,
$$

hence

$$
\omega_{26}=-\omega_{16}, \quad \omega_{14}+2 \omega_{15}+\omega_{25}=0 .
$$

However, $\omega$ is symplectic if and only if $\omega_{16}^{2}\left(\omega_{14}+2 \omega_{15}+\omega_{25}\right) \neq 0$. This is a contradiction.

Suppose that the basis in (d) spans a Lagrangian subspace for a symplectic form as in Lemma 4.19. Then the following pairings of the first and third and second and third basis vector have to vanish:

$$
\omega_{14}-\omega_{15}+b_{5}^{\prime} \omega_{16}-\omega_{15}+\omega_{25}-b_{5}^{\prime} \omega_{26}=0, \quad-\omega_{26}+\omega_{16}=0
$$

hence

$$
\omega_{26}=\omega_{16}, \quad \omega_{14}-2 \omega_{15}+\omega_{25}=0 .
$$

However, $\omega$ is symplectic if and only if $\omega_{16}^{2}\left(\omega_{14}-2 \omega_{15}+\omega_{25}\right) \neq 0$. This is a contradiction.

Proposition 4.21. The Lie algebra $L_{6,17}^{-}$does not admit a bi-Lagrangian structure.

Proof. Suppose that $\mathcal{F}, \mathcal{G}$ is a bi-Lagrangian structure. Considering the bases (a) and (b) in Lemma 4.20, it follows that exactly one of $\mathcal{F}, \mathcal{G}$ must have a basis with a non-zero $e_{1}$-component, say $\mathcal{F}$. Otherwise $\mathcal{F}$ and $\mathcal{G}$ cannot be complementary.

In the both cases $\mathcal{F}$ has a basis of the form

$$
e_{1}+a_{2} e_{2}+a_{3} e_{3}+a_{4} e_{4}+a_{5} e_{5}, \quad b_{4} e_{4}+b_{5} e_{5}, \quad e_{6} .
$$

It follows that $\mathcal{G}$ has a basis of the form

$$
\begin{aligned}
& g_{1}=e_{2}+x_{4} e_{4}+x_{5} e_{5}+x_{6} e_{6} \\
& g_{2}=e_{3}+y_{4} e_{4}+x_{5} e_{5}+y_{6} e_{6} \\
& g_{3}=z_{4} e_{4}+z_{5} e_{5}+z_{6} e_{6} .
\end{aligned}
$$

We get $\left[g_{1},\left[g_{1}, g_{2}\right]\right]=-e_{6} \in \mathcal{F}$. This is a contradiction.

4.1.8. $L_{6,18}, L_{6,19}, L_{6,21}$.

Lemma 4.22. Let $\mathfrak{g}$ be one of the Lie algebras $L_{6,18}, L_{6,19}, L_{6,21}$ and suppose that $\mathfrak{g}$ has two complementary 3 -dimensional subalgebras $\mathcal{F}, \mathcal{G}$. Then one of the two subalgebras, say $\mathcal{F}$, has a basis of the form

$$
e_{1}+\sum_{i=2}^{4} a_{i} e_{i}, \quad e_{5}, \quad e_{6} .
$$

Proof. Suppose that $\mathcal{F}$ has basis vectors $f_{1}, f_{2}$ as in Lemma 4.2 (a).

In $L_{6,18}$ we get $\left[f_{1},\left[f_{1}, f_{2}\right]\right]=b_{2} e_{4}+b_{3} e_{5}+b_{4} e_{6}$. Lemma 2.1 implies that $b_{2}=b_{3}=b_{4}=0$, hence the claim.

In $L_{6,19}$ we get

$$
\left[f_{1},\left[f_{1}, f_{2}\right]\right]=b_{2} e_{4}+b_{3} e_{5}+\left(b_{4}+a_{2} b_{2}\right) e_{6} .
$$

Again we have $b_{2}=b_{3}=b_{4}=0$. 
Finally, in $L_{6,21}$ we get

$$
\left[f_{1},\left[f_{1}, f_{2}\right]\right]=b_{2} e_{4}+\left(b_{3}+a_{2} b_{2}\right) e_{5}+\left(b_{4}+2 a_{2} b_{3}-a_{3} b_{2}\right) e_{6} .
$$

Again $b_{2}=b_{3}=b_{4}=0$.

Proposition 4.23. The Lie algebras $L_{6,18}, L_{6,19}$ and $L_{6,21}$ do not admit a biLagrangian structure.

Proof. Any closed 2-form on $L_{6,18}$ is of the form

$\omega=\omega_{12} \alpha_{12}+\omega_{13} \alpha_{13}+\omega_{23} \alpha_{23}+\omega_{14} \alpha_{14}+\omega_{34} \alpha_{34}+\omega_{15} \alpha_{15}-\omega_{34} \alpha_{25}+\omega_{16} \alpha_{16}$.

Any closed 2-form on $L_{6,19}$ is of the form

$$
\begin{aligned}
\omega= & \omega_{12} \alpha_{12}+\omega_{13} \alpha_{13}+\omega_{23} \alpha_{23}+\omega_{14} \alpha_{14}+\omega_{16} \alpha_{24}+\omega_{34} \alpha_{34}+\omega_{15} \alpha_{15} \\
& -\omega_{34} \alpha_{25}+\omega_{16} \alpha_{16} .
\end{aligned}
$$

Finally, any closed 2-form on $L_{6,21}$ is of the form

$$
\begin{aligned}
\omega= & \omega_{12} \alpha_{12}+\omega_{13} \alpha_{13}+\omega_{23} \alpha_{23}+\omega_{14} \alpha_{14}+\omega_{24} \alpha_{24}+\omega_{34} \alpha_{34}+\omega_{24} \alpha_{15} \\
& +\left(\omega_{16}-\omega_{34}\right) \alpha_{25}+\omega_{16} \alpha_{16} .
\end{aligned}
$$

If the form $\omega$ is symplectic, then in each case $\omega_{16} \neq 0$. It follows that the subalgebra $\mathcal{F}$ in Lemma 4.22 cannot be Lagrangian.

4.2. Calculation of the curvature of the bi-Lagrangian structures in Table 4 , We now calculate the canonical connection and the curvature for the examples of bi-Lagrangian structures in Table 4, see Table 5 for a summary. It turns out that all 16 examples are Ricci-flat (and thus yield para-Kähler analogues of Calabi-Yau manifolds), 8 of which are flat and 8 non-flat.

Remark 4.24. The calculations of the canonical connection can be checked with the statement in Remark 1.6 .

4.2.1. $A_{6}$. A simple calculation shows that the canonical connection $\nabla$ is trivial in the basis $e_{1}, e_{2}, e_{3}, e_{4}, e_{5}, e_{6}$ and $R=0$.

4.2.2. $L_{3} \oplus A_{3}$.

$$
\begin{gathered}
\nabla_{e_{1}} e_{1}=-e_{3}, \quad \nabla_{e_{1}} e_{2}=-e_{6} . \\
R=0 .
\end{gathered}
$$

4.2.3. $L_{5,2} \oplus A_{1}$.

$$
\begin{gathered}
\nabla_{e_{1}} e_{1}=-e_{4}, \quad \nabla_{e_{3}} e_{3}=e_{5}, \quad \nabla_{e_{3}} e_{1}=e_{6}, \quad \nabla_{e_{1}} e_{2}=-e_{5} . \\
R=0 .
\end{gathered}
$$

4.2.4. $L_{3} \oplus L_{3}$.

$$
\begin{gathered}
\nabla_{e_{1}} e_{1}=-e_{4}, \quad \nabla_{e_{3}} e_{3}=e_{2}, \quad \nabla_{e_{3}} e_{4}=-e_{6}, \quad \nabla_{e_{1}} e_{2}=-e_{5} . \\
R\left(e_{1}, e_{3}\right) e_{3}=-e_{5}, \quad R\left(e_{3}, e_{1}\right) e_{1}=e_{6} . \\
\operatorname{Ric}=0 .
\end{gathered}
$$


4.2.5. $L_{6,1}$.

$$
\begin{gathered}
\nabla_{e_{1}} e_{1}=e_{2}, \quad \nabla_{e_{2}} e_{1}=e_{5}, \quad \nabla_{e_{1}} e_{3}=-e_{6}, \quad \nabla_{e_{2}} e_{4}=-e_{6} . \\
R=0 .
\end{gathered}
$$

4.2.6. $L_{6,2}$.

$$
\begin{gathered}
\nabla_{e_{1}} e_{1}=e_{3}, \quad \nabla_{e_{3}} e_{1}=e_{5}, \quad \nabla_{e_{1}+e_{2}}\left(e_{1}+e_{2}\right)=-2 e_{4} \\
\nabla_{e_{4}}\left(e_{1}+e_{2}\right)=-\left(e_{5}-e_{6}\right), \quad \nabla_{e_{4}} e_{1}=e_{5}, \quad \nabla_{e_{1}+e_{2}} e_{3}=-2 e_{5} \\
\nabla_{e_{3}}\left(e_{1}+e_{2}\right)=-\left(e_{5}-e_{6}\right), \quad \nabla_{e_{1}} e_{4}=e_{5}-e_{6} . \\
R\left(e_{1}, e_{1}+e_{2}\right)\left(e_{1}+e_{2}\right)=-2\left(e_{5}-e_{6}\right), \quad R\left(e_{1}+e_{2}, e_{1}\right) e_{1}=-2 e_{5} . \\
\text { Ric }=0 .
\end{gathered}
$$

4.2.7. $L_{5,3} \oplus A_{1}$.

$$
\begin{gathered}
\nabla_{e_{1}} e_{1}=-e_{3}, \quad \nabla_{e_{1}} e_{3}=e_{4}, \quad \nabla_{e_{3}} e_{1}=e_{4} \\
\nabla_{e_{1}} e_{2}=-e_{5}, \quad \nabla_{e_{1}} e_{5}=-e_{6}, \quad \nabla_{e_{3}} e_{2}=e_{6} . \\
R=0 .
\end{gathered}
$$

4.2.8. $L_{6,4}$.

$$
\begin{gathered}
\nabla_{e_{1}} e_{2}=-2 e_{4}, \quad \nabla_{e_{2}} e_{1}=-e_{4}, \quad \nabla_{e_{1}} e_{3}=-e_{5}, \quad \nabla_{e_{2}} e_{3}=-e_{6} . \\
R=0 .
\end{gathered}
$$

4.2.9. $L_{6,5}$.

$$
\begin{gathered}
\nabla_{e_{1}} e_{3}=-e_{5}, \quad \nabla_{e_{1}} e_{1}=e_{3}, \quad \nabla_{e_{1}} e_{2}=-e_{4}, \quad \nabla_{e_{1}} e_{4}=-e_{6} . \\
R=0 .
\end{gathered}
$$

4.2.10. $L_{6,6}$.

$$
\begin{gathered}
\nabla_{e_{1}-e_{2}} e_{3}=-e_{5}, \quad \nabla_{e_{1}-e_{2}}\left(e_{1}-e_{2}\right)=-e_{3}, \quad \nabla_{e_{1}+e_{5}}\left(e_{1}+e_{5}\right)=-e_{4} \\
\nabla_{e_{1}+e_{5}} e_{3}=-e_{5}, \quad \nabla_{e_{1}-e_{2}}\left(e_{1}+e_{5}\right)=-e_{4}, \quad \nabla_{e_{1}-e_{2}} e_{4}=e_{6} . \\
R\left(e_{1}+e_{5}, e_{1}-e_{2}\right)\left(e_{1}-e_{2}\right)=e_{5}, \quad R\left(e_{1}-e_{2}, e_{1}+e_{5}\right)\left(e_{1}+e_{5}\right)=-e_{6} . \\
\operatorname{Ric}=0 .
\end{gathered}
$$

4.2.11. $L_{6,9}$.

$$
\begin{gathered}
\nabla_{e_{1}} e_{3}=-\frac{1}{2} e_{5}, \quad \nabla_{e_{1}} e_{1}=e_{3}, \quad \nabla_{e_{3}} e_{1}=\frac{1}{2} e_{5} \\
\nabla_{e_{3}} e_{2}=e_{6}, \quad \nabla_{e_{1}} e_{2}=-e_{4}, \quad \nabla_{e_{1}} e_{4}=-e_{6} . \\
R=0 .
\end{gathered}
$$

4.2.12. $L_{6,10}$.

$$
\begin{gathered}
\nabla_{e_{4}} e_{1}=e_{5}, \quad \nabla_{e_{2}-e_{4}}\left(e_{3}+e_{5}\right)=-e_{6}, \quad \nabla_{e_{2}-e_{4}}\left(e_{2}-e_{4}\right)=-e_{6} \\
\nabla_{e_{2}-e_{4}} e_{1}=e_{4}-e_{5}, \quad \nabla_{e_{4}}\left(e_{2}-e_{4}\right)=e_{6} . \\
R\left(e_{2}-e_{4}, e_{1}\right) e_{1}=-e_{5}, \quad R\left(e_{1}, e_{2}-e_{4}\right)\left(e_{2}-e_{4}\right)=e_{6} . \\
\operatorname{Ric}=0 .
\end{gathered}
$$


4.2.13. $L_{6,11}$.

$$
\begin{gathered}
\nabla_{e_{1} e_{1}}=e_{4}, \quad \nabla_{e_{4} e_{1}}=e_{5}, \quad \nabla_{e_{1}}\left(e_{3}+e_{5}\right)=-e_{6} \\
\nabla_{e_{1}+e_{2}-e_{4}}\left(e_{3}+e_{5}\right)=-e_{6}, \quad \nabla_{e_{1}+e_{2}-e_{4}} e_{1}=e_{4}-e_{5}, \quad \nabla_{e_{1}+e_{2}-e_{4}} e_{4}=-e_{5} \\
\nabla_{e_{4}}\left(e_{1}+e_{2}-e_{4}\right)=e_{6}, \quad \nabla_{e_{1}+e_{2}-e_{4}}\left(e_{1}+e_{2}-e_{4}\right)=-\left(e_{3}+e_{5}\right)-e_{6} . \\
R\left(e_{1}+e_{2}-e_{4}, e_{1}\right) e_{1}=-2 e_{5}, \quad R\left(e_{1}, e_{1}+e_{2}-e_{4}\right)\left(e_{1}+e_{2}-e_{4}\right)=2 e_{6} . \\
\operatorname{Ric}=0 .
\end{gathered}
$$

4.2.14. $L_{6,12}$.

$$
\begin{gathered}
\nabla_{e_{2}} e_{3}=-7 e_{5}, \quad \nabla_{e_{2} e_{2}}=-\frac{6}{7} e_{3}, \quad \nabla_{e_{3} e_{2}}=-6 e_{5} \\
\nabla_{e_{2}-2 e_{1}}\left(e_{3}-e_{4}\right)=\frac{7}{3}\left(-3 e_{5}+e_{6}\right), \quad \nabla_{e_{2}-2 e_{1}}\left(e_{2}-2 e_{1}\right)=-\frac{10}{7}\left(e_{3}-e_{4}\right) \\
\nabla_{e_{3}-e_{4}}\left(e_{2}-2 e_{1}\right)=\frac{4}{3}\left(-3 e_{5}+e_{6}\right), \quad \nabla_{e_{2}-2 e_{1}} e_{2}=2 e_{3} \\
\nabla_{e_{3}-e_{4}} e_{2}=4 e_{5}, \quad \nabla_{e_{2}-2 e_{1}} e_{3}=5 e_{5}, \quad \nabla_{e_{2}}\left(e_{2}-2 e_{1}\right)=2\left(e_{3}-e_{4}\right) \\
\nabla_{e_{3}}\left(e_{2}-2 e_{1}\right)=-2\left(-3 e_{5}+e_{6}\right), \quad \nabla_{e_{2}}\left(e_{3}-e_{4}\right)=-\left(-3 e_{5}+e_{6}\right) . \\
R\left(e_{2}-2 e_{1}, e_{2}\right) e_{2}=\frac{208}{7} e_{5} \\
R\left(e_{2}, e_{2}-2 e_{1}\right)\left(e_{2}-2 e_{1}\right)=-\frac{208}{21}\left(-3 e_{5}+e_{6}\right) . \\
\text { Ric }=0 .
\end{gathered}
$$

4.2.15. $L_{5,4} \oplus A_{1}$.

$$
\begin{gathered}
\nabla_{e_{1}} e_{5}=-e_{6}, \quad \nabla_{e_{4}} e_{4}=-e_{3}, \quad \nabla_{e_{4}} e_{1}=e_{5}, \quad \nabla_{e_{1}} e_{2}=-e_{4} . \\
R\left(e_{4}, e_{1}\right) e_{1}=e_{6}, \quad R\left(e_{1}, e_{4}\right) e_{2}=-e_{3} \\
R\left(e_{2}, e_{1}\right) e_{1}=-e_{5}, \quad R\left(e_{1}, e_{2}\right) e_{4}=-e_{3} . \\
\operatorname{Ric}=0 .
\end{gathered}
$$

4.2.16. $L_{6,16}$.

$$
\begin{array}{cc}
\nabla_{e_{1}} e_{4}=-e_{6}, \quad \nabla_{e_{3}} e_{3}=-e_{5}, & \nabla_{e_{3}} e_{2}=e_{5} \\
\nabla_{e_{3}} e_{1}=e_{4}, \quad \nabla_{e_{1}} e_{2}=-e_{3} . \\
R\left(e_{3}, e_{1}\right) e_{1}=e_{6}, \quad R\left(e_{1}, e_{3}\right) e_{2}=-e_{5}, \quad R\left(e_{1}, e_{2}\right) e_{3}=-e_{5} \\
R\left(e_{1}, e_{2}\right) e_{2}=e_{5}, \quad R\left(e_{2}, e_{1}\right) e_{1}=-e_{4} . \\
\text { Ric }=0 .
\end{array}
$$

5. APPENDIX 


\begin{tabular}{|c|cc|c|c|}
\hline Algebraic structure & \multicolumn{2}{|c|}{ Structure constants } & Symplectic form & Bi-Lagrangian structure \\
\hline & $d \alpha_{3}$ & $d \alpha_{4}$ & & \\
\hline \hline$A_{4}$ & 0 & 0 & $\alpha_{12}+\alpha_{34}$ & $\left\{e_{1}, e_{3}\right\},\left\{e_{2}, e_{4}\right\}$ \\
\hline$L_{3} \oplus A_{1}\left(\mathfrak{n i l}_{3} \oplus \mathbb{R}\right)$ & 0 & $\alpha_{12}$ & $\alpha_{14}+\alpha_{23}$ & $\left\{e_{1}, e_{3}\right\},\left\{e_{2}, e_{4}\right\}$ \\
\hline$L_{4}\left(\mathfrak{n i l}_{4}\right)$ & $\alpha_{12}$ & $\alpha_{13}$ & $\alpha_{14}+\alpha_{23}$ & not bi-Lagrangian \\
\hline
\end{tabular}

TABLE 1. 4-dimensional nilpotent Lie algebras $\left(d \alpha_{i}=0\right.$ for $\left.i=1,2\right)$ 


\begin{tabular}{|c|c|c|c|c|}
\hline \multicolumn{2}{|c|}{ Betti numbers } & \multicolumn{3}{|c|}{ Algebraic structure } \\
\hline$b_{1}$ & $b_{2}$ & Salamon & Khakimdjanov et al. & Bazzoni-Muñoz \\
\hline 6 & 15 & $(0,0,0,0,0,0)$ & 26 & $A_{6}$ \\
\hline 5 & 11 & $(0,0,0,0,0,12)$ & 25 & $L_{3} \oplus A_{3}$ \\
\hline 4 & 9 & $(0,0,0,0,12,13)$ & 23 & $L_{5,2} \oplus A_{1}$ \\
\hline 4 & 8 & $(0,0,0,0,12,34)$ & 24 & $L_{3} \oplus L_{3}$ \\
\hline 4 & 8 & $(0,0,0,0,12,14+23)$ & 17 & $L_{6,1}$ \\
\hline 4 & 8 & $(0,0,0,0,13+42,14+23)$ & 16 & $L_{6,2}$ \\
\hline 4 & 7 & $(0,0,0,0,12,15)$ & 22 & $L_{4} \oplus A_{2}$ \\
\hline 4 & 7 & $(0,0,0,0,12,14+25)$ & 21 & $L_{5,3} \oplus A_{1}$ \\
\hline 3 & 8 & $(0,0,0,12,13,23)$ & 18 & $L_{6,4}$ \\
\hline 3 & 6 & $(0,0,0,12,13,14)$ & 14 & $L_{6,5}$ \\
\hline 3 & 6 & $(0,0,0,12,13,24)$ & 15 & $L_{6,6}$ \\
\hline 3 & 6 & $(0,0,0,12,13,14+23)$ & 13 & $L_{6,9}$ \\
\hline 3 & 5 & $(0,0,0,12,13+14,24)$ & 11 & $L_{6,10}$ \\
\hline 3 & 5 & $(0,0,0,12,14,13+42)$ & 10 & $L_{6,11}$ \\
\hline 3 & 5 & $(0,0,0,12,13+42,14+23)$ & 12 & $L_{6,12}$ \\
\hline 3 & 5 & $(0,0,0,12,14,15)$ & 19 & $L_{5,4} \oplus A_{1}$ \\
\hline 3 & 5 & $(0,0,0,12,14,15+23)$ & 9 & $L_{6,13}$ \\
\hline 3 & 5 & $(0,0,0,12,14,15+24)$ & 20 & $L_{5,6} \oplus A_{1}$ \\
\hline 3 & 5 & $(0,0,0,12,14,15+23+24)$ & 7 & $L_{6,14}$ \\
\hline 3 & 4 & $(0,0,0,12,14-23,15+34)$ & 8 & $L_{6,15}$ \\
\hline 2 & 4 & $(0,0,12,13,23,14)$ & 6 & $L_{6,16}$ \\
\hline 2 & 4 & $(0,0,12,13,23,14+25)$ & 4 & $L_{6,17}^{+}$ \\
\hline 2 & 4 & $(0,0,12,13,23,14-25)$ & 5 & $L_{6,17}^{-}$ \\
\hline 2 & 3 & $(0,0,12,13,14,15)$ & 3 & $L_{6,18}$ \\
\hline 2 & 3 & $(0,0,12,13,14,23+15)$ & 2 & $L_{6,19}$ \\
\hline 2 & 3 & $(0,0,12,13,14+23,24+15)$ & 1 & $L_{6,21}$ \\
\hline
\end{tabular}

TABLE 2. Comparison of notation for 6 -dimensional symplectic nilpotent Lie algebras in [19], [16] and [3] 


\begin{tabular}{|c|c|c|c|c|}
\hline \multirow{2}{*}{ Algebraic structure } & \multicolumn{4}{|c|}{ Structure constants } \\
\hline & $d \alpha_{3}$ & $d \alpha_{4}$ & $d \alpha_{5}$ & $d \alpha_{6}$ \\
\hline$A_{6}$ & 0 & 0 & 0 & 0 \\
\hline$L_{3} \oplus A_{3}$ & 0 & 0 & 0 & $\alpha_{12}$ \\
\hline$L_{5,2} \oplus A_{1}$ & 0 & 0 & $\alpha_{12}$ & $\alpha_{13}$ \\
\hline$L_{3} \oplus L_{3}$ & 0 & 0 & $\alpha_{12}$ & $\alpha_{34}$ \\
\hline$L_{6,1}$ & 0 & 0 & $\alpha_{12}$ & $\alpha_{13}+\alpha_{24}$ \\
\hline$L_{6,2}$ & 0 & 0 & $\alpha_{13}-\alpha_{24}$ & $\alpha_{14}+\alpha_{23}$ \\
\hline$L_{4} \oplus A_{2}$ & 0 & 0 & $\alpha_{12}$ & $\alpha_{15}$ \\
\hline$L_{5,3} \oplus A_{1}$ & 0 & 0 & $\alpha_{12}$ & $\alpha_{15}+\alpha_{23}$ \\
\hline$L_{6,4}$ & 0 & $\alpha_{12}$ & $\alpha_{13}$ & $\alpha_{23}$ \\
\hline$L_{6,5}$ & 0 & $\alpha_{12}$ & $\alpha_{13}$ & $\alpha_{14}$ \\
\hline$L_{6,6}$ & 0 & $\alpha_{12}$ & $\alpha_{13}$ & $\alpha_{24}$ \\
\hline$L_{6,9}$ & 0 & $\alpha_{12}$ & $\alpha_{13}$ & $\alpha_{14}+\alpha_{23}$ \\
\hline$L_{6,10}$ & 0 & $\alpha_{12}$ & $\alpha_{14}$ & $\alpha_{23}+\alpha_{24}$ \\
\hline$L_{6,11}$ & 0 & $\alpha_{12}$ & $\alpha_{14}$ & $\alpha_{13}+\alpha_{24}$ \\
\hline$L_{6,12}$ & 0 & $\alpha_{12}$ & $\alpha_{14}+\alpha_{23}$ & $\alpha_{13}-\alpha_{24}$ \\
\hline$L_{5,4} \oplus A_{1}$ & 0 & $\alpha_{12}$ & $\alpha_{14}$ & $\alpha_{15}$ \\
\hline$L_{6,13}$ & 0 & $\alpha_{12}$ & $\alpha_{14}$ & $\alpha_{15}+\alpha_{23}$ \\
\hline$L_{5,6} \oplus A_{1}$ & 0 & $\alpha_{12}$ & $\alpha_{14}$ & $\alpha_{15}+\alpha_{24}$ \\
\hline$L_{6,14}$ & 0 & $\alpha_{12}$ & $\alpha_{14}$ & $\alpha_{15}+\alpha_{23}+\alpha_{24}$ \\
\hline$L_{6,15}$ & 0 & $\alpha_{12}$ & $\alpha_{14}+\alpha_{23}$ & $\alpha_{15}-\alpha_{34}$ \\
\hline$L_{6,16}$ & $\alpha_{12}$ & $\alpha_{13}$ & $\alpha_{23}$ & $\alpha_{14}$ \\
\hline$L_{6,17}^{+}$ & $\alpha_{12}$ & $\alpha_{13}$ & $\alpha_{23}$ & $\alpha_{14}+\alpha_{25}$ \\
\hline$L_{6,17}^{-}$ & $\alpha_{12}$ & $\alpha_{13}$ & $\alpha_{23}$ & $\alpha_{14}-\alpha_{25}$ \\
\hline$L_{6,18}$ & $\alpha_{12}$ & $\alpha_{13}$ & $\alpha_{14}$ & $\alpha_{15}$ \\
\hline$L_{6,19}$ & $\alpha_{12}$ & $\alpha_{13}$ & $\alpha_{14}$ & $\alpha_{15}+\alpha_{23}$ \\
\hline$L_{6,21}$ & $\alpha_{12}$ & $\alpha_{13}$ & $\alpha_{14}+\alpha_{23}$ & $\alpha_{15}+\alpha_{24}$ \\
\hline
\end{tabular}

TABLE 3. Structure constants $\left(d \alpha_{i}=0\right.$ for $\left.i=1,2\right)$ 


\begin{tabular}{|c|c|c|}
\hline Algebraic structure & Symplectic form & Bi-Lagrangian structure \\
\hline$A_{6}$ & $\alpha_{12}+\alpha_{34}+\alpha_{56}(\mathrm{BM})$ & $\left\{e_{1}, e_{3}, e_{5}\right\},\left\{e_{2}, e_{4}, e_{6}\right\}$ \\
\hline$L_{3} \oplus A_{3}$ & $\alpha_{16}+\alpha_{23}+\alpha_{45}(\mathrm{BM})$ & $\left\{e_{1}, e_{3}, e_{4}\right\},\left\{e_{2}, e_{5}, e_{6}\right\}$ \\
\hline$L_{5,2} \oplus A_{1}$ & $\alpha_{15}+\alpha_{24}+\alpha_{36}(\mathrm{BM})$ & $\left\{e_{1}, e_{4}, e_{6}\right\},\left\{e_{2}, e_{3}, e_{5}\right\}$ \\
\hline$L_{3} \oplus L_{3}$ & $\alpha_{15}+\alpha_{24}+\alpha_{36}(\mathrm{BM})$ & $\left\{e_{1}, e_{4}, e_{6}\right\},\left\{e_{2}, e_{3}, e_{5}\right\}$ \\
\hline$L_{6,1}$ & $\alpha_{16}+\alpha_{23}-\alpha_{45}(\mathrm{~K})$ & $\left\{e_{1}, e_{2}, e_{5}\right\},\left\{e_{3}, e_{4}, e_{6}\right\}$ \\
\hline$L_{6,2}$ & $\alpha_{16}+\alpha_{25}+\alpha_{34}(\mathrm{BM})$ & $\left\{e_{1}, e_{3}, e_{5}\right\},\left\{e_{1}+e_{2}, e_{4}, e_{5}-e_{6}\right\}$ \\
\hline$L_{4} \oplus A_{2}$ & $\alpha_{16}+\alpha_{25}+\alpha_{34}(\mathrm{BM})$ & not bi-Lagrangian \\
\hline$L_{5,3} \oplus A_{1}$ & $\alpha_{16}+\alpha_{24}-\alpha_{35}(\mathrm{BM})$ & $\left\{e_{1}, e_{3}, e_{4}\right\},\left\{e_{2}, e_{5}, e_{6}\right\}$ \\
\hline$L_{6,4}$ & $\alpha_{16}+2 \alpha_{25}+\alpha_{34}(\mathrm{~K})$ & $\left\{e_{1}, e_{2}, e_{4}\right\},\left\{e_{3}, e_{5}, e_{6}\right\}$ \\
\hline$L_{6,5}$ & $\alpha_{16}+\alpha_{25}+\alpha_{34}(\mathrm{~K})$ & $\left\{e_{1}, e_{3}, e_{5}\right\},\left\{e_{2}, e_{4}, e_{6}\right\}$ \\
\hline$L_{6,6}$ & $\alpha_{15}+\alpha_{25}-\alpha_{26}+\alpha_{34}(\mathrm{~K})$ & $\left\{e_{1}-e_{2}, e_{3}, e_{5}\right\},\left\{e_{1}+e_{5}, e_{4}, e_{6}\right\}$ \\
\hline$L_{6,9}$ & $\alpha_{16}+2 \alpha_{25}+\alpha_{34}(\mathrm{BM})$ & $\left\{e_{1}, e_{3}, e_{5}\right\},\left\{e_{2}, e_{4}, e_{6}\right\}$ \\
\hline$L_{6,10}$ & $\alpha_{16}+\alpha_{25}-\alpha_{34}(\mathrm{BM})$ & $\left\{e_{1}, e_{4}, e_{5}\right\},\left\{e_{2}-e_{4}, e_{3}+e_{5}, e_{6}\right\}$ \\
\hline$L_{6,11}$ & $\alpha_{16}+\alpha_{25}-\alpha_{26}-\alpha_{34}(\mathrm{~K})$ & $\left\{e_{1}, e_{4}, e_{5}\right\},\left\{e_{1}+e_{2}-e_{4}, e_{3}+e_{5}, e_{6}\right\}$ \\
\hline$L_{6,12}$ & $-\alpha_{15}+6 \alpha_{26}+7 \alpha_{34}(\mathrm{~K})$ & $\left\{e_{2}, e_{3}, e_{5}\right\},\left\{e_{2}-2 e_{1}, e_{3}-e_{4},-3 e_{5}+e_{6}\right\}$ \\
\hline$L_{5,4} \oplus A_{1}$ & $\alpha_{13}+\alpha_{26}-\alpha_{45}(\mathrm{BM})$ & $\left\{e_{1}, e_{5}, e_{6}\right\},\left\{e_{2}, e_{3}, e_{4}\right\}$ \\
\hline$L_{6,13}$ & $\alpha_{13}+\alpha_{26}-\alpha_{45}(\mathrm{BM})$ & not bi-Lagrangian \\
\hline$L_{5,6} \oplus A_{1}$ & $\alpha_{13}+\alpha_{26}-\alpha_{45}(\mathrm{BM})$ & not bi-Lagrangian \\
\hline$L_{6,14}$ & $\alpha_{13}+\alpha_{26}-\alpha_{45}(\mathrm{BM})$ & not bi-Lagrangian \\
\hline$L_{6,15}$ & $\alpha_{16}+\alpha_{24}-\alpha_{35}(\mathrm{~K})$ & not bi-Lagrangian \\
\hline$L_{6,16}$ & $\alpha_{15}+\alpha_{24}+\alpha_{26}-\alpha_{34}(\mathrm{~K})$ & $\left\{e_{1}, e_{4}, e_{6}\right\},\left\{e_{2}, e_{3}, e_{5}\right\}$ \\
\hline$L_{6,17}^{+}$ & $\alpha_{16}+\alpha_{15}+\alpha_{24}+\alpha_{35}(\mathrm{BM})$ & not bi-Lagrangian \\
\hline$L_{6,17}^{-}$ & $\alpha_{15}-\alpha_{16}+\alpha_{24}+\alpha_{35}(\mathrm{~K})$ & not bi-Lagrangian \\
\hline$L_{6,18}$ & $\alpha_{16}+\alpha_{25}-\alpha_{34}(\mathrm{BM})$ & not bi-Lagrangian \\
\hline$L_{6,19}$ & $\alpha_{16}+\alpha_{24}+\alpha_{25}-\alpha_{34}(\mathrm{BM})$ & not bi-Lagrangian \\
\hline$L_{6,21}$ & $2 \alpha_{16}+\alpha_{25}+\alpha_{34}(\mathrm{BM})$ & not bi-Lagrangian \\
\hline
\end{tabular}

TABLE 4. Symplectic and bi-Lagrangian structures ((BM) and (K) indicate that the symplectic form is taken from [3] and [16], respectively; note Remark 4.1) 


\begin{tabular}{|c|c|}
\hline Algebraic structure & Curvature tensor $R$ \\
\hline \hline$A_{6}$ & 0 \\
\hline$L_{3} \oplus A_{3}$ & 0 \\
\hline$L_{5,2} \oplus A_{1}$ & $\neq 0$ \\
\hline$L_{3} \oplus L_{3}$ & 0 \\
\hline$L_{6,1}$ & $\neq 0$ \\
\hline$L_{6,2}$ & 0 \\
\hline$L_{5,3} \oplus A_{1}$ & 0 \\
\hline$L_{6,4}$ & 0 \\
\hline$L_{6,5}$ & $\neq 0$ \\
\hline$L_{6,6}$ & 0 \\
\hline$L_{6,9}$ & $\neq 0$ \\
\hline$L_{6,10}$ & $\neq 0$ \\
\hline$L_{6,11}$ & $\neq 0$ \\
\hline$L_{6,12}$ & $\neq 0$ \\
\hline$L_{5,4} \oplus A_{1}$ & $\neq 0$ \\
\hline$L_{6,16}$ & $\neq 0$ \\
\hline$C u r v a t u r e f b$ & $\neq 0$ \\
\hline
\end{tabular}

TABLE 5. Curvature of bi-Lagrangian structures in Table 4 (all examples are Ricci-flat) 
Acknowledgements. My work on this topic dates back to my diploma thesis [11] at the LMU Munich. I am grateful to D. Kotschick for supervising the thesis and for many helpful discussions and suggestions in the meantime. I also want to thank the anonymous referee for helpful comments.

\section{REFERENCES}

1. C. Bai, Left-symmetric bialgebras and an analogue of the classical Yang-Baxter equation, Commun. Contemp. Math. 10 (2008), no. 2, 221-260.

2. O. Baues, V. Cortés, Symplectic Lie groups, Astérisque 379, Société Mathématique de France 2016.

3. G. Bazzoni, V. Muñoz, Classification of minimal algebras over any field up to dimension 6, Trans. Amer. Math. Soc. 364 (2012), no. 2, 1007-1028.

4. S. Benayadi, M. Boucetta, On para-Kähler and hyper-para-Kähler Lie algebras, J. Algebra 436 (2015), 61-101.

5. N. B. Boyom, Varietes symplectiques affines, Manuscripta Math. 64 (1989), 1-33.

6. D. Conti, F. A. Rossi, The Ricci tensor of almost parahermitian manifolds, Ann. Global Anal. Geom. 53 (2018), no. 4, 467-501.

7. D. Conti, S. Salamon, Generalized Killing spinors in dimension 5, Trans. Amer. Math. Soc. 359 (2007), no. 11, 5319-5343.

8. V. Cruceanu, P. Fortuny, P. M. Gadea, A survey on paracomplex geometry, Rocky Mountain J. Math. 26 (1996), no. 1, 83-115.

9. F. Etayo Gordejuela, R. Santamaría, The canonical connection of a bi-Lagrangian manifold, Journ. of Phys. A 34 (2001), 981-987.

10. D. Giovannini, Special structures and symplectic geometry, Ph.D. thesis, Università degli Studi di Torino, 2003.

11. M. J. D. Hamilton, Bi-Lagrangian structures on closed manifolds, Diplomarbeit, LMU München 2004, 84 pp.

12. M. J. D. Hamilton, D. Kotschick, Künneth geometry, book manuscript.

13. H. Hess, Connections on symplectic manifolds and geometric quantization, Lect. Notes in Math. 836 (1980), 153-166.

14. N. J. Hitchin, Hypersymplectic quotients, in La "Mécanique analytique" de Lagrange et son héritage, Supplemento al numero 124 (1990) degli Atti della Accademia delle Scienze di Torino, Classe de Scienze Fisiche, Matematiche e Naturali, 169-180.

15. S. Kaneyuki, Homogeneous symplectic manifolds and dipolarizations in Lie algebras, Tokyo J. Math. 15 (1992), no. 2, 313-325.

16. Y. Khakimdjanov, M. Goze, A. Medina, Symplectic or contact structures on Lie groups, Differential Geom. Appl. 21 (2004), no. 1, 41-54.

17. A. I. Malcev, On a class of homogeneous spaces, AMS Transl. 9 (Series 1) (1962) 276-307.

18. F. A. Rossi, D-complex structures: cohomological properties and deformations, Ph.D. thesis, Università degli Studi di Milano-Bicocca (2013). http://hdl.handle.net/10281/41976

19. S. M. Salamon, Complex structures on nilpotent Lie algebras, J. Pure Appl. Algebra 157 (2001), no. 2-3, 311-333.

Fachbereich Mathematik, Universität Stuttgart, Pfaffenwaldring 57, 70569 STUTTGART, GERMANY

E-mail address: mark. hamilton@math. lmu.de 